\title{
Experimental demonstration of a laser proton accelerator with accurate beam control through image-relaying transport
}

\author{
J. G. Zhu, ${ }^{1}$ M. J. Wu, ${ }^{1}$ Q. Liao, ${ }^{1}$ Y. X. Geng, ${ }^{1}$ K. Zhu,,${ }^{1}$ C. C. Li, ${ }^{1}$ X. H. Xu,${ }^{1}$ D. Y. Li,,${ }^{1}$ Y. R. Shou, ${ }^{1}$ \\ T. Yang, ${ }^{1}$ P. J. Wang, ${ }^{1}$ D. H. Wang, ${ }^{1}$ J. J. Wang, ${ }^{4}$ C. E. Chen, ${ }^{1}$ X. T. He, ${ }^{1}$ Y. Y. Zhao, ${ }^{1}$ W. J. Ma, ${ }^{1}$ \\ H. Y. Lu, ${ }^{1,3}$ T. Tajima, ${ }^{5}$ C. Lin, ${ }^{1,3, \dagger}$ and X. Q. Yan ${ }^{1,2,3, *}$ \\ ${ }^{1}$ State Key Laboratory of Nuclear Physics and Technology, and Key Laboratory of HEDP of the Ministry \\ of Education, CAPT, Peking University, Beijing 100871, China \\ ${ }^{2}$ Peking University Shenzhen Graduate School, Shenzhen, Guangdong 518055, China \\ ${ }^{3}$ Collaborative Innovation Center of Extreme Optics, Shanxi University, Shanxi 030006, China \\ ${ }^{4}$ Beijing Third Hospital, Peking University, Beijing 100191, China \\ ${ }^{5}$ Department of Physics and Astronomy, UC Irvine, Irvine, California 92697, USA
}

(Received 21 February 2019; published 24 June 2019)

\begin{abstract}
A compact laser plasma accelerator (CLAPA) that can stably produce and transport proton ions with different energies less than $10 \mathrm{MeV},<1 \%$ energy spread, several to tens of $\mathrm{pC}$ charge, is demonstrated. The high current proton beam with continuous energy spectrum and a large divergence angle is generated by using a high contrast laser and micron thickness targets, which later is collected, analyzed and refocused by an image-relaying beam line using a combination of quadrupole and bending electromagnets. It eliminates the inherent defects of the laser-driven beams, realizes precise manipulation of the proton beams with reliability, availability, maintainability and inspectability (RAMI), and takes the first step towards applications of this new generation of accelerator. With the development of high-rep rate Petawatt (PW) laser technology, we can now envision a new generation of accelerator for many applications in the near future soon.
\end{abstract}

DOI: 10.1103/PhysRevAccelBeams.22.061302

\section{INTRODUCTION}

The concept of laser acceleration was first invented by Tajima and Dawson [1] in 1979, and was soon extended from accelerating electrons using gas targets to accelerating ions using solid targets. When an ultrahigh-intensity laser irradiates on a solid target, the established acceleration field can exceed $\mathrm{TV} / \mathrm{m}$, accelerating ions to relativistic energy within tens of micron distance. It can significantly shrink down the size and the cost of the traditional radio frequency accelerators. The laser-driven high-current ion sources may enable significant advances in tumor therapy [2], radiograph for high density physics [3], isotope generation for positron emission tomography [4], warm dense matter [5] and fast ignition of fusion cores [6]. However, many scientific and technical challenges for laser acceleration

\footnotetext{
*orresponding author. x.yan@pku.edu.cn

Corresponding author. lc0812@pku.edu.cn

Published by the American Physical Society under the terms of the Creative Commons Attribution 4.0 International license. Further distribution of this work must maintain attribution to the author(s) and the published article's title, journal citation, and DOI.
}

need to be overcome before a real laser accelerator with reliable operations can be built and applied to these applications.

For proton accelerations, target normal sheath acceleration (TNSA) $[7,8]$ and radiation pressure acceleration (RPA) [9-15] or breakout afterburner (BOA) [16] are the most widely employed mechanisms. In TNSA mechanisms, the acceleration field is established by laserproduced thermal electrons, which is simple and reliable. The proton energy is proportional to the square root of laser intensity and the maximum proton energy of $85 \mathrm{MeV}$ was achieved by using a huge laser system with $\sim 100 \mathrm{~J}$ per shot [17]. Nanometer thin foils are used in RPA or BOA regimes for bulk acceleration and near $100 \mathrm{MeV}$ proton beams were demonstrated $[18,19]$. As a high contrast laser pulse and ultrahigh laser intensity are necessary for the RPA/BOA regime, therefore so far quite few results are reported. Although significant progress has been made in this field, argument also exists that the laser accelerator will not be able to replace conventional ones any time soon because of the proton energy, flux, shot-to-shot reproducibility, accurate dose control, and so on [20]. For example, the typical exponentially decaying spectrum of laser-accelerated protons is far from performance levels for many applications. A few experiments have demonstrated monoenergetic proton beams using microstructured target [21,22] or 
gaseous target $[23,24]$, but with poor reproducibility or energy flexibility. To implement the application of the laser accelerated protons, massive efforts have to be made to solve the broad energy spread, the large divergence angle, the poor reproducibility and flexibility, which are necessary conditions to raise "laser acceleration" to "laser accelerator."

To grapple with these problems of laser-driven proton beams, at first step, permanent magnet quadrupole lenses [25-27], solenoid magnets [28-30] and laser-triggered microlenses [31] are demonstrated as the focusing components. Later on, various beam lines have been proposed at several institutes. For instance, the light beam line at GSI Helmholtz Center has demonstrated a multi-MeV proton beam with high peak intensity, subnanosecond pulse duration [32] and improved homogeneity [33]. The ELIMED beam line installed in Prague uses magnet chicane as the energy selection unit, aiming to deliver controllable proton beam up to $60 \mathrm{MeV}$ for therapeutic purposes [34]. Masood proposed a compact gantry design with pulsed magnets for the laser-driven proton radiotherapy [35]. However, a complete magnet beam line which propagates the high current and dense proton beam with $1 \%$ energy spread and with reliability, availability, maintainability and inspectability (RAMI) has never been reported in any experiment yet. Laser accelerated ion beam normally has a high peak current, broadband energy spectrum and large divergence angle, which are the bottlenecks for beam transport. Furthermore, laser-accelerated ion beam may contain energy-space correlation, which must be considered.

To overcome these obstacles thoroughly, a beam line consisting of quadrupole and bending electromagnets has been designed and built at Peking University. Comparing with the beam lines that use quadrupole sets [36] or chicane of dipoles $[34,37]$ for energy manipulation, the object-toimage point analyzing system can realize the separation of protons with different divergence angles and energies, ensuring the accuracy of proton beam energy selection. Here we report the realization of laser-driven proton beam of different energies less than $10 \mathrm{MeV}$ with $1 \%$ energy spread using image-relaying transport beam line. The proton beam parameters, such as energy, energy bandwidth, uniformity and diameter are precisely controlled. Although the proton energy demonstrated here is still far from meeting the requirements of some applications, such as cancer therapy, realizing precise manipulation of the monoenergetic proton beams with RAMI is still an important step, which lays the foundation for subsequent experiments, such as precise biological dose deposition, space irradiation environment simulation, measurement of energy stopping in warm dense matter, detection device calibration and measurement of proton beam parameters.

\section{EXPERIMENT SETUP}

The experiments were carried out on a compact laser plasma accelerator (CLAPA) platform at Peking University, as shown in Fig. 2. The whole platform is built on a three meter thick reinforced concrete foundation to ensure the stability of the system. The p-polarized laser pulse with $1.8 \mathrm{~J}$ energy and $30 \mathrm{fs}$ duration was focused onto the $1.2 \mu \mathrm{m}$ thickness plastic target using an $f / 3.5$ off axis parabola at an incident angle of 30 degrees with respect to the target normal direction. The spot diameter (FWHM) of the laser was $5 \mu \mathrm{m}$ with $25 \%$ of total energy, corresponding to an intensity of $8 \times 10^{19} \mathrm{~W} / \mathrm{cm}^{2}$ on target. The laser contrast was $10^{-10}$ at 40 ps before the main pulse using an cross-polarized wave generation (XPW), which ensured the effective acceleration. A high magnification imaging system was used to ensure the accurate coupling between the laser focal spot and each target, with a spatial resolution of 0.1 micrometer. A Thomson spectrometer, coupled with a microchannel plate (MCP) and electron-multiplying charge-coupled devices (EMCCD), is placed at $14 \mathrm{~cm}$ behind the target to measure the energy spectrum of the protons. Figure 1(a) shows the typical energy spectrum measured by the Thomson spectrometer (black curve). During the beam line experiment, this Thomson spectrometer was replaced by a quadrupole triplet lens. Then an insertable radiochromic film (RCF) stack positioned $4 \mathrm{~cm}$ behind the target was used to measure the original spatial and energy distribution of the protons. Figure 1(b) shows typical images of one RCF stack based on a $1.2 \mu \mathrm{m}$ plastic target, where three types of gafchromic film (HD-V2, MD-V3, EBT-3) were used due to the exponential decay of the particle numbers from low energy to high energy.

(a) Energy spectrum of the proton beam

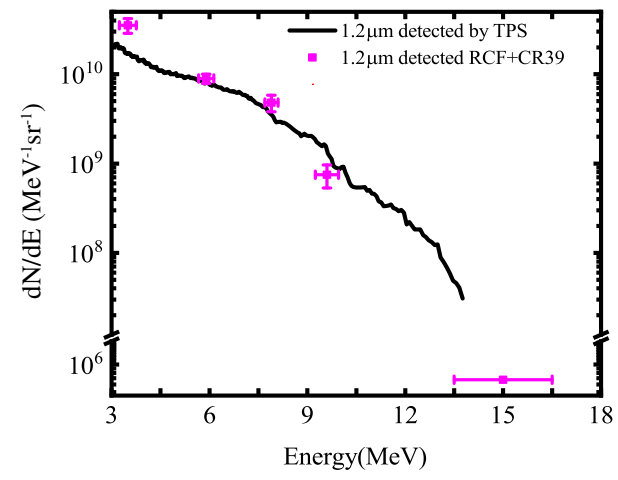

(b) Proton beam distributions on RCF stacks

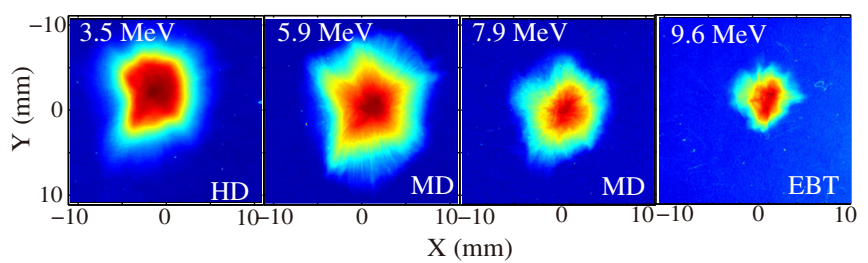

FIG. 1. (a) The typical energy spectrum measured by the Thomson spectrometer (black curve) and the RCF stack (red dots) based on a $1.2 \mu$ m plastic target. (b) Images in one RCF stack, where three types of gafchromic film (HD-V2, MD-V3, EBT-3) were used. 
The corresponding energy spectrum extracted from the RCF stack is shown in Fig. 1(a) (red dots), which is quite consistent with the Thomson spectrometer measurement.

\section{PROTON BEAM TRANSPORT}

\section{A. Generation of monoenergetic proton beam}

A magnet lattice consisting of quadrupole and bending electromagnets is specially designed to deliver laser-driven proton beam with energy of 1-15 MeV, as shown in Fig. 2. The protons are first collected and focused by a quadrupole triplet lens placed $19 \mathrm{~cm}$ behind the target with a collection angle of $\pm 50 \mathrm{mrad}$, then analyzed by a 45 -degree sector magnet, and finally refocused by a quadrupole doublet lens onto the irradiation platform. The parameters of each magnetic component are shown in Table I. For the selected beam energy, the focal point of the quadrupole triplet lens overlaps with the object point (Slit\#1) of the sector magnet, whose image point (Slit\#2) is $20 \mathrm{~cm}$ in front of the quadrupole doublet. The beam line incorporates three beam profile detectors (the first two using scintillators, the third using MCP), located near the focal plane of the triplet lens, the image point of the sector magnet and at the irradiation point respectively (labeled BPD\#1, BPD\#2 and BPD\#3 respectively in Fig. 2). The focal plane of the triplet lens can be precisely adjusted onto BPD\#1 or onto the first slit, and the image point of the sector magnet can be adjusted onto BPD\#2 or onto the second slit for switching between beam measurement and transport. This flexibility is crucial to enable effective beam diagnostics without impacting applications.

The inserted graph in the top left in Fig. 2 shows the simulated envelope evolution of proton beam along the beam line using the TRACK program. The initial beam is set with $10 \mu \mathrm{m}$ diameter, $100 \mathrm{mrad}$ divergence angle and energy of $15 \mathrm{MeV}$ with $\pm 1 \%$ energy spread (consistent with the cutoff energy measured by CR39). Part of the beam is blocked in the triplet lens due to the aperture limitation. As the quadrupole triplet lens is placed $19 \mathrm{~cm}$ behind the target to make room for laser/target/proton measurements, the collection efficiency of the triplet lens is about $88 \%$ for protons within $\pm 50 \mathrm{mrad}$. After the triplet lens, the transport efficiency of the beam with $\pm 1 \%$ energy spread is nearly $100 \%$.

Figures 3(a) and 3(b) are the simulated and experimental results of the proton beam profile on the BPD\#1 after focused by triplet lens. The focused central energy is set at $5 \mathrm{MeV}$. Slit\#1 is put at the object point of the bending magnet, which can partially screen the unwanted energies, leading to about $\pm 10 \%$ energy spread of the beam within $3 \mathrm{~cm}^{2}$ transverse area. One can note that the beam spot distribution at BPD\#1 has a cross shape with blurred boundary, due to the chromatic aberration and large acceptance angle of the initial beam.

Following this, a 45-degree sector magnet is used to select the energy at the image point in the $x$ direction (horizontal direction). This is an object-to-image point transport system, meaning protons from the same object point with the same energy and different angular divergence converge to the same

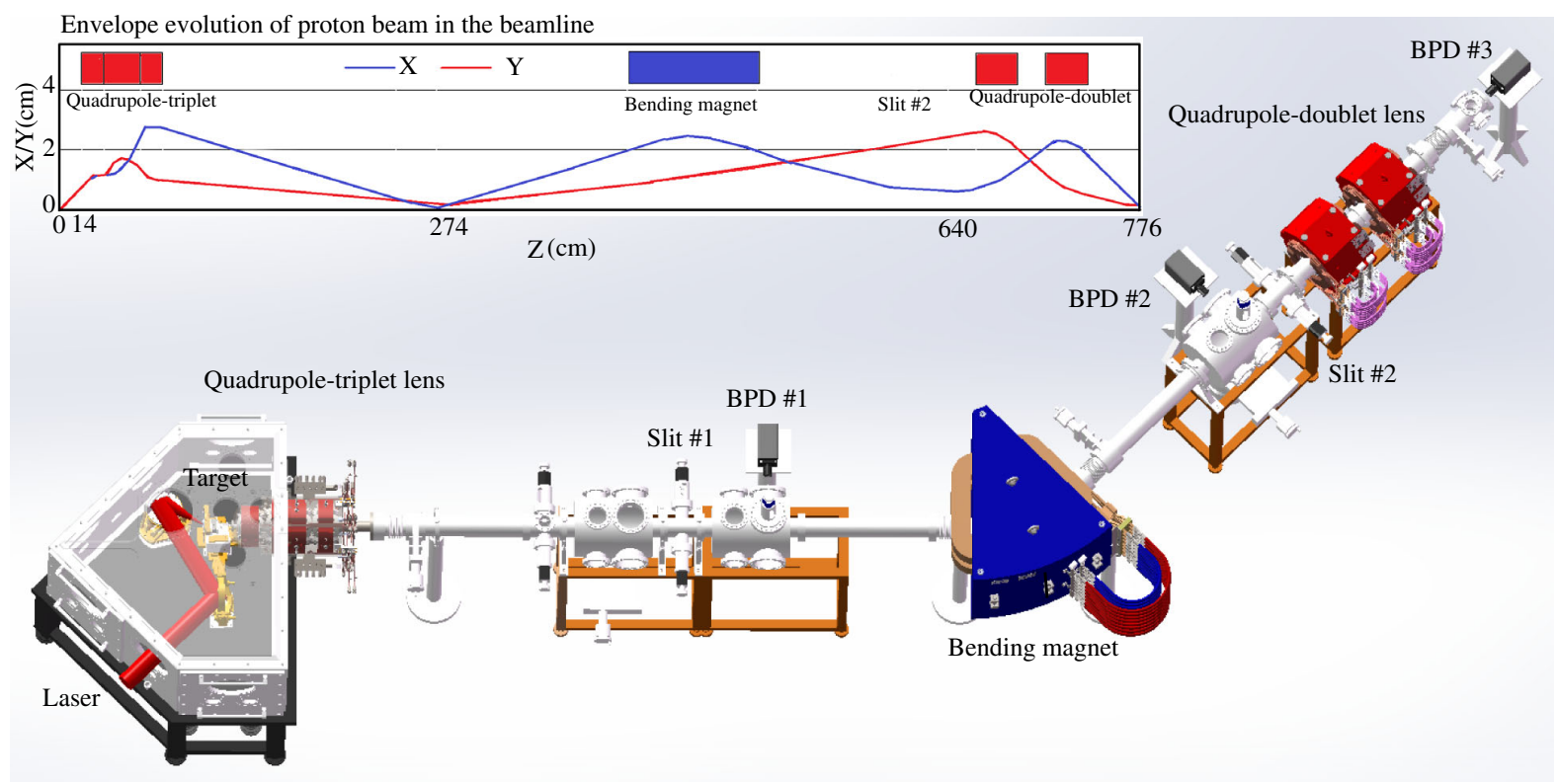

FIG. 2. Layout of compact laser plasma accelerator based on a high power laser. The proton beam is accelerated by using a high contrast laser and micro-thickness foil; it is then focused by a quadrupole triplet lens, analyzed by a bending electromagnet and refocused by a quadrupole doublet lens with selected energy onto the irradiation platform. The inserted graph in the top left is the simulated envelope evolution of the proton beam with a central energy of $15 \mathrm{MeV}$. $X$ is the horizontal direction and $Y$ is the vertical direction. The radius of the initial beam spot is set as $5 \mu \mathrm{m}$ and the acceptance angle is $\pm 50 \mathrm{mrad}$. 
TABLE I. The CLAPA beam line parameters.

\begin{tabular}{lccccc}
\hline \hline Type & Length & Aperture & Max B & \# turns & Current \\
\hline Q1 & $100 \mathrm{~mm}$ & $30 \mathrm{~mm}$ & $5 \mathrm{KGs} / \mathrm{cm}$ & 16 & $300 \mathrm{~A}$ \\
Q2 & $200 \mathrm{~mm}$ & $64 \mathrm{~mm}$ & $2.5 \mathrm{KGs} / \mathrm{cm}$ & 20 & $540 \mathrm{~A}$ \\
Q3 & $100 \mathrm{~mm}$ & $64 \mathrm{~mm}$ & $2.5 \mathrm{KGs} / \mathrm{cm}$ & 20 & $540 \mathrm{~A}$ \\
B1 & $511 \mathrm{~mm}$ & $70 \mathrm{~mm}(\mathrm{gap})$ & $1.5 \mathrm{~T}$ & 64 & $850 \mathrm{~A}$ \\
Q4 & $300 \mathrm{~mm}$ & $110 \mathrm{~mm}$ & $0.727 \mathrm{KGs} / \mathrm{cm}$ & 44 & $220 \mathrm{~A}$ \\
Q5 & $300 \mathrm{~mm}$ & $110 \mathrm{~mm}$ & $0.727 \mathrm{KGs} / \mathrm{cm}$ & 44 & $220 \mathrm{~A}$ \\
\hline \hline
\end{tabular}

image point at the $x$ axis, while protons with different energies are separated in the $x$ direction. This means that the sector magnet, together with the triplet lens, can efficiently capture and analyze the protons generated in laser acceleration, and the influences of large divergence angle, large energy spread and angular-dependent distribution of initial protons are removed at the image point. In this system energy chirp does not impact the transverse distribution of protons. Figures 3(c) and 3(d) are the simulated and the experimental spatial distributions of the proton beam at the BPD\#2. They both show a bow tie profile with $5 \mathrm{MeV}$ protons at the knot, which indicates the chromatic aberration in the $y$ direction, and the ones with energies deviated from
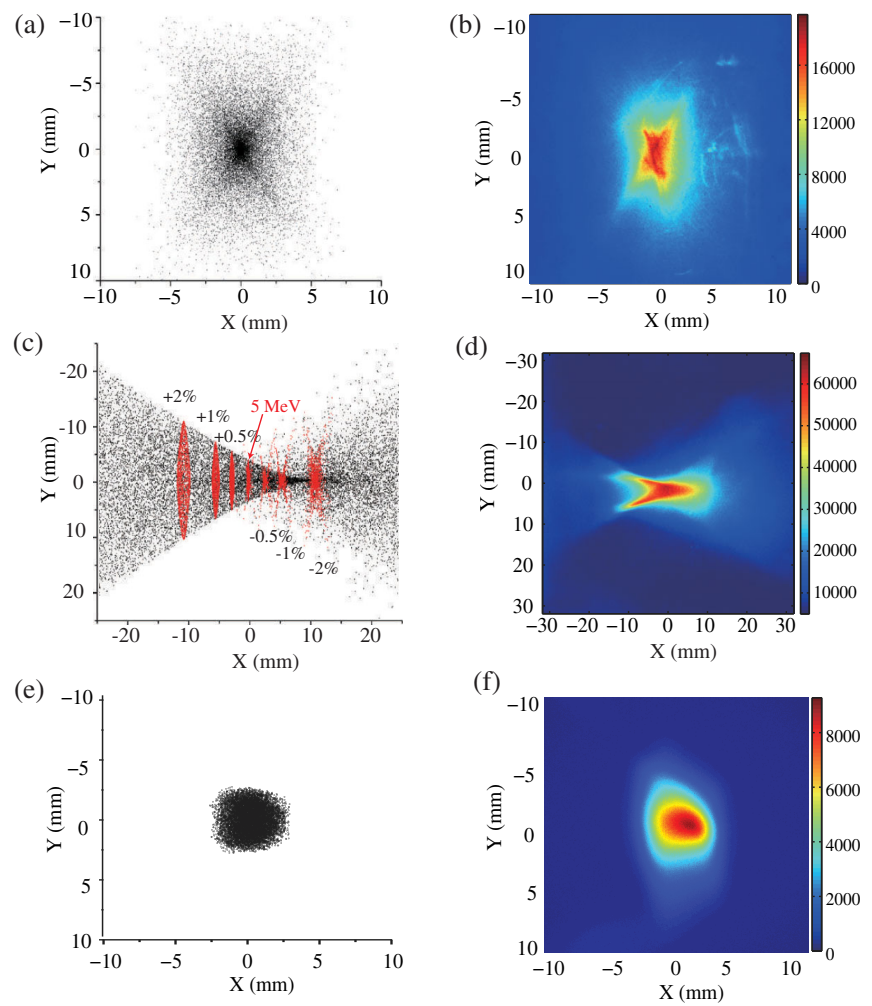

FIG. 3. Parts (a), (c), (e) and (b), (d), (f) are the simulated and experimental results of the proton beam profile on BPD\#1, BPD\#2 and BPD\#3, respectively. The selected proton energy is $5 \mathrm{MeV}$ and the energy spread is $\pm 1 \%$. The red dots in (c) from right to left represent protons with energy deviated $-2 \%,-1 \%$, $-0.5 \%, 0 \%, 0.5 \%, 1 \%$ and $2 \%$ from $5 \mathrm{MeV}$ respectively.
$5 \mathrm{MeV}$ are dispersed aside. For example, beam with $\pm 2 \%$ energy spread is dispersed to $\pm 11 \mathrm{~mm}$ while beam with $\pm 1 \%$ energy spread is dispersed to $\pm 5.5 \mathrm{~mm}$. So by controlling the opening width of slit\#2, which is installed at the image point of the sector magnet, the energy spread of protons can be precisely controlled.

In the last, a quadrupole doublet lens is used to focus the monoenergetic protons to the irradiation point with desired spot size. Figures 3(e) and 3(f) show the simulated and experimental spatial distributions of the proton beam at the BPD\#3 (5 MeV and $\pm 1 \%$ energy spread). The final focused beam profile can be adjusted as required, for the quadrupole doublet lens has the advantages of controlling envelopes independently in the $x$ and the $y$ direction.

The energy spectrum evolution of the proton beam through the beam line is presented in Fig. 4, which demonstrates how the initial broadband beam energy is gradually confined to $\pm 1 \%$ energy spread after each electromagnet. The black curve is the original energy spectrum deconvolved from the RCF data shown in Fig. 1(b), by using a Monte Carlo ion transport code SRIM26. The black dashed curve is the energy spectrum entering the beam line, taking into account the $\pm 50 \mathrm{mrad}$ acceptance angle. The green, purple, blue and red curves are the simulated spectra, respectively, at BPD\#1, BPD\#2 and BPD\#3 (the irradiation platform). In the experiment, by varying the width of Slit\#2 from 3 to $54 \mathrm{~mm}$ (the corresponding energy spread is increased from $\pm 0.5 \%$ to $\pm 4 \%$ ), and the final charge was tuned correspondingly.

\section{B. Adjustment of the beam line}

To show tunability of this CLAPA beam line, by fixing the opening width of Slit\#2 at $14 \mathrm{~mm}$, and by fine adjusting the current of the electromagnets, proton beam with different central energies and $\pm 1 \%$ energy spread were transmitted and focused onto BPD\#3. Figure 5 shows how the measured proton charge on BPD\#3 varies with energy. As the proton energy increased from 3 to $9 \mathrm{MeV}$, the charge

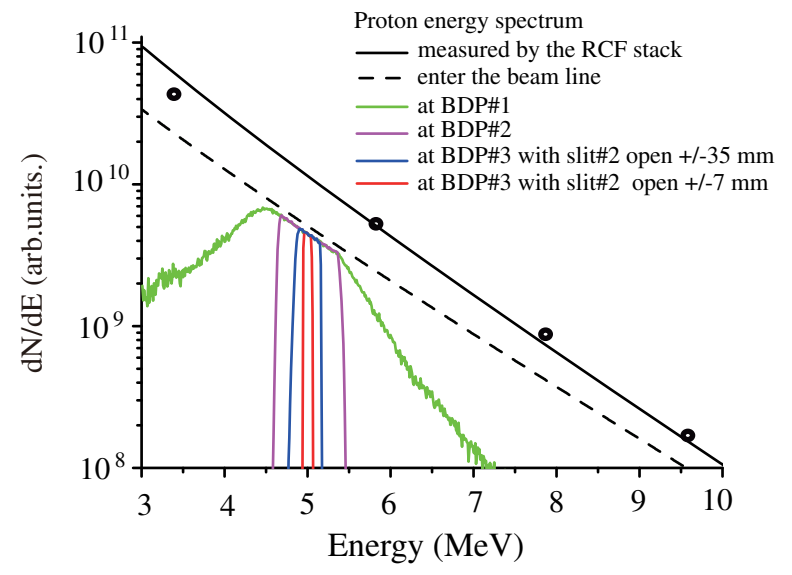

FIG. 4. Evolution of the proton energy spectra along the beam line. The central energy is $5 \mathrm{MeV}$. 


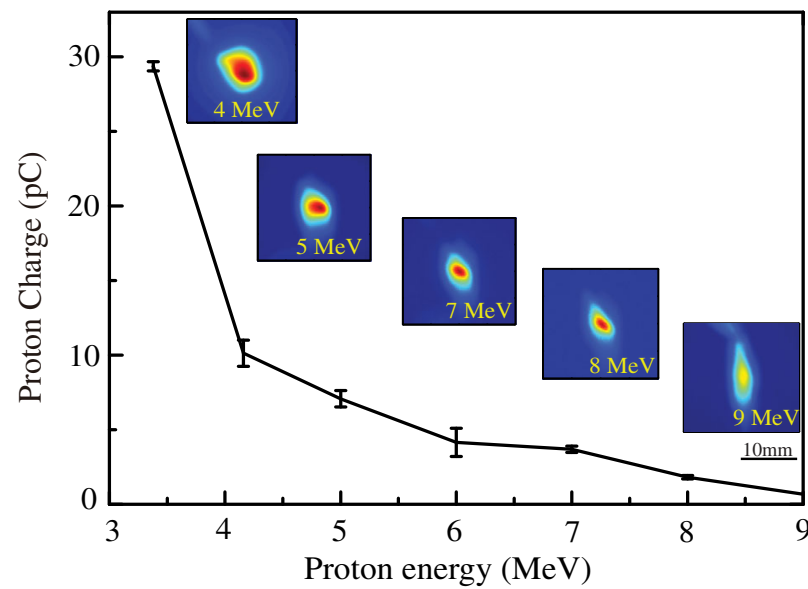

FIG. 5. Scaling of the proton charge with different central energies on the irradiation platform (BPD\#3). The inserted pictures show beam profiles at different energies. The slit width is $14 \mathrm{~mm}$ and the corresponding energy spread is $\pm 1 \%$.

was reduced from 30 to $0.2 \mathrm{pC}$. The more than 2 orders of magnitude reduction is consistent with the RCF measurement. The charge and the spot size of the nine consecutive shots of protons with $7 \mathrm{MeV}$ central energy and $\pm 1 \%$ energy spread are shown in Fig. 6. The averaged charge is $2.8 \mathrm{pC}$ with $8 \%$ charge rms deviation and the averaged diameter is $3.4 \mathrm{~mm}$ with $11 \% \mathrm{rms}$ deviation. The stability can be further improved by increasing the quality of the laser pulse, the uniformity of the solid target, and the space coupling of the laser with the target.

Besides charge and energy tunability, a uniform beam profile is also necessary for many applications. For example, dose distribution of protons in organic tissue will be uneven, and the effectiveness of cancer therapies will be unreliable if the proton distribution is malformed. By manipulating the beam waist position in the $y$ direction, radially symmetric proton distributions at the irradiation platform are realized [38]. In the experiments of transporting $5 \mathrm{MeV}$ proton beams with $\pm 1 \%$ energy spread onto the irradiation platform, by adjusting the magnetic fields, (a)

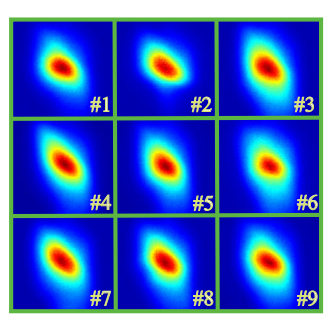

(b)

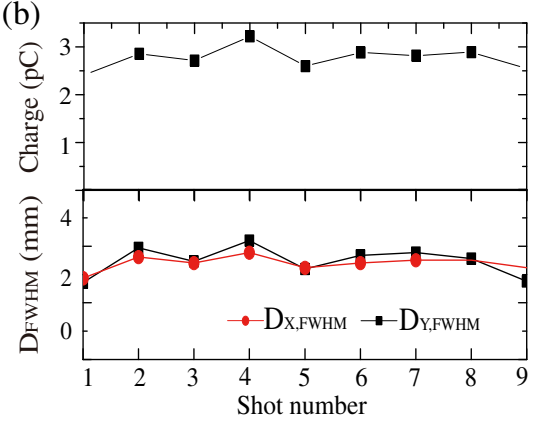

FIG. 6. (a) Repeatability of proton beams on the irradiation platform with $7 \mathrm{MeV}$ central energy and $\pm 1 \%$ energy spread for nine shots in series. (b) The corresponding fluctuations of the proton charge and spot diameter.
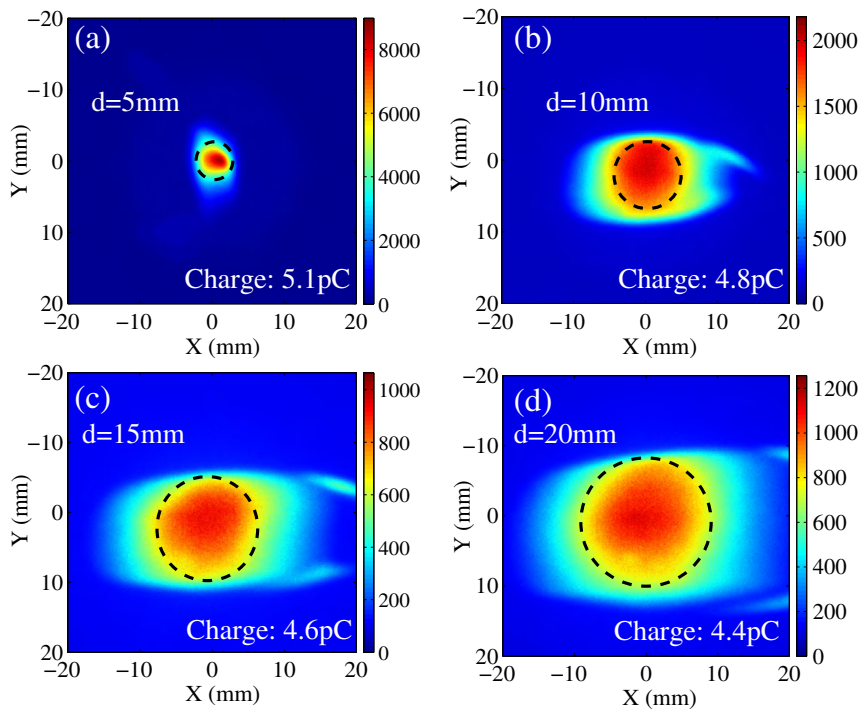

FIG. 7. Demonstration of the spot size adjustment of $5 \mathrm{MeV}$ proton beam with $\pm 1 \%$ energy spread refocused at the irradiation point. The diameter of the black dashed circle is from $5 \mathrm{~mm}$ in (a) to $20 \mathrm{~mm}$ in (d).

the beam spot can be regulated with different sizes and shapes, as marked by the black dashed circles, while maintaining radially symmetric distributions, as shown in Fig. 7. So with an extra central aperture (or mask), radially symmetric monoenergetic pencil beams can be obtained by laser accelerator without extra beam scattering equipment. This ability is very important for expanding the application field of laser accelerator.

\section{SUMMARY}

In summary, the CLAPA experimental results show that the laser plasma accelerator integrated with a magnet lattice can reliably deliver protons with beam qualities suitable for many applications, such as $1 \%$ energy spread of different energies and good uniformity. The experiment demonstrates precise adjustment of the laser accelerated proton beam in terms of energy, charge and diameter with repeatability and availability. It raises the laser acceleration to laser accelerator of $\sim 10 \mathrm{MeV}$ protons through beam control since the invention of laser acceleration in 1979. The setup of sector magnet properly integrated with triplet and doublet quadrupole lenses can overcome inherent drawbacks of the laser-driven beams, and paves the way for laser accelerator in a wide range of applications. With the radially symmetric monoenergetic beams demonstrated at CLAPA, primary biomedical cell irradiation experiments and material irradiation experiments to emulate the space conditions are under way. With the development of highrep rate Petawatt (PW) laser technology [39], the proton energy and current will be soon available for the applications such as cancer therapy. This image-relaying technology can be easily applied to the $200-230 \mathrm{MeV}$ high energy 
protons, resorting to pulsed magnets or superconducting magnets. It is hoped that a compact beam therapeutic machine of cancer treatment based on laser accelerator can be developed in the near future.

\section{ACKNOWLEDGMENTS}

This work was supported by National Basic Research Program of China (Grant No. 2013CBA01502), National Natural Science Foundation of China (Grants No. 11475010, No. 11575011, No. 11535001, No. 11775010, and No. 61631001), ITER-CHINA Program (Grant No. 2015GB120001), and National Grand Instrument Project (2012YQ030142).

J. G. Z., M. J. W. and Q. L. made an equal contribution to this work in experiments.

[1] T. Tajima and J. Dawson, Laser Electron Accelerator, Phys. Rev. Lett. 43, 267 (1979).

[2] C. M. Ma, I. Veltchev, E. Fourkal, J. S. Li, W. Luo, J. Fan, T. Lin, and A. Pollack, Development of a laser-driven proton accelerator for cancer therapy, Laser Phys. 16, 639 (2006).

[3] J. R. Rygg, F. H. Séguin, C. K. Li, J. A. Frenje, M. J.-E. Manuel, R. D. Petrasso et al., Proton radiography of inertial fusion implosions, Science 319, 1223 (2008).

[4] I. Spencer, K. W. D. Ledingham, R. P. Singhal, T. Mccanny, P. McKenna, E. L. Clark, K. Krushelnick et al., Laser generation of proton beams for the production of short-lived positron emitting radioisotopes, Nucl. Instrum. Methods Phys. Res., Sect. B 183, 449 (2001).

[5] P. K. Patel, A. J. Mackinnon, M. H. Key, T. E. Cowan, M. E. Foord, M. Allen, D. F. Price, H. Ruhl, P. T. Springer, and R. Stephens, Isochoric Heating of Solid-Density Matter with an Ultrafast Proton Beam, Phys. Rev. Lett. 91, 125004 (2003).

[6] M. Roth, T. E. Cowan, M. H. Key, S. P. Hatchett, C. Brown, W. Fountain, J. Johnson et al., Fast Ignition by Intense Laser-Accelerated Proton Beams, Phys. Rev. Lett. 86, 436 (2001).

[7] B. M. Hegelich, B. J. Albright, J. Cobble, K. Flippo, S. Letzring, M. Paffett, H. Ruhl, J. Schreiber, R. K. Schulze, and J. C. Fernández, Laser acceleration of quasimonoenergetic $\mathrm{MeV}$ ion beams, Nature (London) 439, 441 (2006).

[8] J. Fuchs, P. Antici, E. d'Humières, E. Lefebvre, M. Borghesi, E. Brambrink, C. A. Cecchetti et al., Laserdriven proton scaling laws and new paths towards energy increase, Nat. Phys. 2, 48 (2006).

[9] T. Esirkepov, M. Borghesi, S. V. Bulanov, G. Mourou, and T. Tajima, Highly Efficient Relativistic-Ion Generation in the Laser-Piston Regime, Phys. Rev. Lett. 92, 175003 (2004).

[10] A. Macchi, F. Cattani, T. V. Liseykina, and F. Cornolti, Laser Acceleration of Ion Bunches at the Front Surface of Overdense Plasmas, Phys. Rev. Lett. 94, 165003 (2005).
[11] A. Macchi, M. Borghesi, and M. Passoni, Ion acceleration by superintense laser-plasma interaction, Rev. Mod. Phys. 85, 751 (2013).

[12] S. M. Weng, Z. M. Sheng, M. Murakami, M. Chen, M. Liu et al., Optimization of hole-boring radiation pressure acceleration of ion beams for fusion ignition, Matter Radiat. Extremes 3, 28 (2018).

[13] V. K. Tripathi, Tung-Chang Liu, and X. Shao, Laser radiation pressure proton acceleration in gaseous target, Matter Radiat. Extremes 2, 256 (2017).

[14] X. Q. Yan, C. Lin, Z. M. Sheng, Z. Y. Guo, B. C. Liu, Y. R. Lu, J. X. Fang, and J. E. Chen, Generating High-Current Monoenergetic Proton Beams by a Circularly Polarized Laser Pulse in the Phase-Stable Acceleration Regime, Phys. Rev. Lett. 100, 135003 (2008).

[15] S. Kar, K. F. Kakolee, B. Qiao, A. Macchi, M. Cerchez, D. Doria, M. Geissler et al., Ion Acceleration in Multispecies Targets Driven by Intense Laser Radiation Pressure, Phys. Rev. Lett. 109, 185006 (2012).

[16] L. Yin, B. J. Albright, K. J. Bowers, D. Jung, J. C. Fernández, and B. M. Hegelich, Three-Dimensional Dynamics of Breakout Afterburner Ion Acceleration Using High-Contrast Short-Pulse Laser and Nanoscale Targets, Phys. Rev. Lett. 107, 045003 (2011).

[17] F. Wagner, O. Deppert, C. Brabetz, P. Fiala, A. Kleinschmidt, P. Poth, V. A. Schanz et al., Maximum Proton Energy Above $85 \mathrm{MeV}$ from the Relativistic Interaction of Laser Pulses with Micrometer Thick $\mathrm{CH}_{2}$ Targets, Phys. Rev. Lett. 116, 205002 (2016).

[18] I. J. Kim, K. H. Pae, I. W. Choi, C. L. Lee, H. T. Kim, H. Singhal, J. H. Sung, S. K. Lee, H. W. Lee, P. V. Nickles, T. M. Jeong, C. M. Kim, and C. H. Nam, Radiation pressure acceleration of protons to $93 \mathrm{MeV}$ with circularly polarized petawatt laser pulses, Phys. Plasmas 23, 070701 (2016).

[19] A. Higginson, R. J. Gray, M. King, R. J. Dance, S. D. R. Williamson, N. M. H. Butler et al., Near-100 MeV protons via a laser-driven transparency-enhanced hybrid acceleration scheme, Nat. Commun. 9, 724 (2018).

[20] U. Linz and J. Alonso, Laser-driven ion accelerators for tumor therapy revised, Phys. Rev. Accel. Beams 19, 124802 (2016).

[21] H. Schwoerer, S. Pfotenhauer, O. Jäckel, K.-U. Amthor, B. Liesfeld, W. Ziegler, R. Sauerbrey, K. W. D. Ledingham, and T. Esirkepov, Laser-plasma acceleration of quasimonoenergetic protons from microstructured targets, Nature (London) 439, 445 (2006).

[22] S. M. Pfotenhauer, O. Jäckel, A. Sachtleben, J. Polz, W. Ziegler, H. P. Schlenvoigt, K. U. Amthor et al., Spectral shaping of laser generated proton beams, New J. Phys. 10, 033034 (2008).

[23] C. A. Palmer, N. P. Dover, I. Pogorelsky, M. Babzien, G. I. Dudnikova, M. Ispiriyan, M. N. Polyanskiy et al., Monoenergetic Proton Beams Accelerated by a Radiation Pressure Driven Shock, Phys. Rev. Lett. 106, 014801 (2011).

[24] D. Haberberger, S. Tochitsky, F. Fiuza, C. Gong, R. A. Fonseca, L. O. Silva, W. B. Mori, and C. Joshi, Collisionless shocks in laser-produced plasma generate monoenergetic high-energy proton beams, Nat. Phys. 8, 95 (2012). 
[25] M. Nishiuchi, I. Daito, M. Ikegami, H. Daido, M. Mori, S. Orimo, K. Ogura et al., Focusing and spectral enhancement of a repetition-rated, laser-driven, divergent multi-mev proton beam using permanent quadrupole magnets, Appl. Phys. Lett. 94, 061107 (2009).

[26] M. Schollmeier, S. Becker, M. Geißel, K. A. Flippo, A. Blažević, S. A. Gaillard, D. C. Gautier et al., Controlled Transport and Focusing of Laser-Accelerated Protons with Miniature Magnetic Devices, Phys. Rev. Lett. 101, 055004 (2008).

[27] L. Pommarel, B. Vauzour, F. Mégnin-Chanet, E. Bayart, O. Delmas, F. Goudjil, C. Nauraye et al., Spectral and spatial shaping of a laser-produced ion beam for radiation-biology experiments, Phys. Rev. Accel. Beams 20, 032801 (2017).

[28] I. Hofmann, J. Meyer-ter-Vehn, X. Q. Yan, A. Orzhekhovskaya, and S. Yaramyshev, Collection and focusing of laser accelerated ion beams for therapy applications, Phys. Rev. ST Accel. Beams 14, 031304 (2011).

[29] I. Hofmann, Performance of solenoids versus quadrupoles in focusing and energy selection of laser accelerated protons, Phys. Rev. ST Accel. Beams 16, 041302 (2013).

[30] K. Harres, I. Alber, A. Tauschwitz, V. Bagnoud, H. Daido, M. Günther, F. Nürnberg, A. Otten, M. Schollmeier, J. Schütrumpf, M. Tampo, and M. Roth, Beam collimation and transport of quasineutral laser-accelerated protons by a solenoid field, Phys. Plasmas 17, 023107 (2010).

[31] T. Toncian, M. Borghesi, J. Fuchs, E. d'Humières, P. Antici, P. Audebert, E. Brambrink, C. A. Cecchetti, A. Pipahl, L. Romagnani, and O. Willi, Ultrafast laser-driven microlens to focus and energy-select mega-electron volt protons, Science 312, 410 (2006).

[32] S. Busold, D. Schumacher, C. Brabetz, D. Jahn, F. Kroll, O. Deppert et al., Towards highest peak intensities for ultrashort MeV-range ion bunches, Sci. Rep. 5, 12459 (2015).

[33] D. Jahn, D. Schumacher, C. Brabetz, J. Ding, S. Weih, F. Kroll, F. E. Brack, U. Schramm, A. Blažević, and M. Roth, First application studies at the laser-driven LIGHT beam line: Improving proton beam homogeneity and imaging of a solid target, Nucl. Instrum. Methods Phys. Res., Sect. A 909, 173 (2018).

[34] F. Romano, F. Schillaci, G. A. P. Cirrone, G. Cuttone, V. Scuderi, L. Allegra et al., The ELIMED transport and dosimetry beam line for laser-driven ion beams, Nucl. Instrum. Methods Phys. Res., Sect. A 829, 153 (2016).

[35] U. Masood, T. E. Cowan, W. Enghardt, K. M. Hofmann, L. Karsch, F. Kroll, U. Schramm, J. J. Wilkens, and J. Pawelke, A light-weight compact proton gantry design with a novel dose delivery system for broad-energetic laser-accelerated beams, Phys. Med. Biol. 62, 5531 (2017).

[36] F. Schillaci, L. Pommarel, F. Romano, G. Cuttone, M. Costa, D. Giove, M. Maggiore et al., Characterization of the ELIMED permanent magnets quadrupole system prototype with laser-driven proton beams, J. Instrum. 11, T07005 (2016).

[37] G. Milluzzo, J. Pipek, A. G. Amico, G. A. P. Cirrone, G. Cuttone, G. Korn, G. Larosa et al., Geant4 simulation of the ELIMED transport and dosimetry beam line for highenergy laser-driven ion beam multidisciplinary applications, Nucl. Instrum. Methods Phys. Res., Sect. A 909, 298 (2018).

[38] J. G. Zhu, K. Zhu, L. Tao, X. H. Xu, C. Lin, W. J. Ma et al., Distribution uniformity of laser-accelerated proton beams, Chin. Phys. C 41, 097001 (2017).

[39] G. Mourou, B. Bill, T. Toshiki, and J. Limpert, The future is fiber accelerators, Nat. Photonics 7, 258 (2013). 